\title{
miR-145 inhibits breast cancer cell growth through RTKN
}

\author{
SHIHUA WANG, CHUNJING BIAN, ZHUO YANG, YE BO, JING LI, \\ LIFEN ZENG, HONG ZHOU and ROBERT CHUNHUA ZHAO
}

Center of Tissue Engineering, Institute of Basic Medical Sciences, Chinese Academy of Medical Sciences, School of Basic Medicine Peking Union Medical College, Beijing 100005, P.R. China

Received November 25, 2008; Accepted February 3, 2009

DOI: 10.3892/ijo_00000275

\begin{abstract}
MicroRNAs (miRNAs) represent a class of small non-coding RNAs regulating gene expression by inducing RNA degradation or interfering with translation. Aberrant miRNA expression has been described for several human malignancies. Herein, we show that miR-145 is down-regulated in human cancer cell line MCF-7 when compared to normal human mammary epithelial cell line MCF10A. Overexpression of miR-145 by plasmid inhibits MCF-7 cell growth and induces apoptosis. Subsequently, RTKN is identified as a potential miR-145 target by bioinformatics. Using reporter constructs, we show that the RTKN 3' untranslated region (3'UTR) carries the directly binding site of miR-145. Additionally, overexpression of miR-145 in MCF-7 reduces RTKN protein expression as well as mRNA level. Furthermore, downregulation of RTKN by siRNA can inhibit MCF-7 cell growth Taken together, we propose that loss of miR-145 may provide a selective growth advantage for MCF-7 by targeting RTKN.
\end{abstract}

\section{Introduction}

MicroRNAs are small, non-coding RNAs that regulate gene expression by targeting the 3' untranslated region (3'UTR) of mRNAs, inducing RNA degradation or interfering with translation (1). This class of regulatory transcripts has diverse functions, including the regulation of cellular differentiation, proliferation, and apoptosis (2). Hence, deregulation of miRNA expression may lead to a variety of disorders, including human cancer. Emerging evidence indicates that many microRNAs are deregulated in human malignancies and it has been proposed that some microRNAs may have oncogenic or tumor suppressor functions (3). Accordingly, both inactivation and overexpression of specific microRNAs have been described in a number of cancer types (4-6). For instance,

Correspondence to: Professor Robert Chunhua Zhao, Center of Tissue Engineering, Institute of Basic Medical Sciences, Chinese Academy of Medical Sciences, School of Basic Medicine Peking Union Medical College, Beijing 100005, P.R. China

E-mail: chunhuaz@public.tpt.tj.cn

Key words: microRNA, miR-145, growth inhibition, RTKN
miR-15a and miR-16 are down-regulated by hemizygous or homozygous deletion or other unknown mechanisms in $68 \%$ of CLLs (7) and miR-17-92 cluster is markedly overexpressed in B-cell lymphomas (8). Also in a large-scale analysis of 540 tumor samples from lung, breast, stomach, prostate, colon, and pancreatic tumors, a so-called solid cancer microRNA signature was identified (9). However, although miRNAs have been the subject of extensive research in recent years, the molecular basis of miRNA-mediated gene regulation and the effect of these genes on tumor growth remain largely unknown because of our limited understanding of miRNA target genes.

Breast cancer is the second leading cause of cancer related deaths among women in the US (10). Recently, some miRNAs have been reported to be associated with the initiation and progression of breast cancer $(11,12)$. In this study, we found that miR-145 was down-regulated in human breast tumor cell line MCF7. Importantly, overexpression of miR-145 suppressed MCF-7 cell growth and induced apoptosis in vitro. In MCF-7 cells, we confirmed that RTKN was a target of miR-145. RTKN (Rhotekin) was initially isolated as a scaffold protein interacting with GTP bound form of Rho (13). It links the Rho signal to nuclear factor- $\mathrm{\kappa B}(\mathrm{NF}-\kappa \mathrm{B})$ activation, leading to increased cell survival by transactivating antiapoptotic genes downstream of NF-кB (14). We found that miR-145 was able to modulate RTKN expression by directly targeting the binding site within the 3'UTR. Elevated levels of miR-145 repressed the cellular mRNA and protein levels of RTKN. Furthermore, down-regulation of RTKN by siRNA can inhibit MCF-7 cell growth. Taken together, we found that miR-145 could inhibit MCF-7 cell growth by targeting RTKN.

\section{Materials and methods}

Cell culture and transfection. Human breast cancer MCF-7 cells were cultured in Dulbecco's modified Eagle's medium (DMEM, Gibco Life Technologies) containing 10\% heatinactivated fetal bovine serum (FBS, Gibco Life Technologies), $100 \mathrm{U} / \mathrm{ml}$ penicillin, and $100 \mu \mathrm{g} / \mathrm{ml}$ streptomycin in a humidified incubator of $5 \% \mathrm{CO}_{2}$ at $37^{\circ} \mathrm{C}$. Transfection of MCF-7 cells was performed with Lipofectamine 2000 (Invitrogen) following the manufacturer's protocol. siRNA (sense, 5'-AGC AUC AGU AAC CAG UAU GTT-3'; and antisense, 5'-CAU ACU GGU UAC UGA UGC UTT-3') that target RTKN was synthesized by Sigma. Transfections were performed in triplicate for each treatment. 
Semi-quantitative RT-PCR. For the detection of protein-coding genes, $1 \mu \mathrm{g}$ of total RNA extracted from cells was reverse transcribed to cDNA primed by oligo(dT) using M-MLV reverse transcriptase (Promega). The cDNA was used for the amplification of RTKN genes and an endogenous control gene GAPDH through reaction. PCR primers were: RTKN sense, 5'-AAAGGTGCTGGCATAGGATCTGC-3'; and RTKN antisense, 5'-TGGTTGATGTGGGAGTCACAA-3'. GAPDH sense, 5'-GAAGGTGAAGGTCGGAGTC-3'; and GAPDH antisense, 5'-GAAGATGGTGATGGGATTTC-3'. PCR cycles were as follows: initial denaturation at $95^{\circ} \mathrm{C}$ for $5 \mathrm{~min}$, followed by 35 cycles of $94^{\circ} \mathrm{C}$ for $1 \mathrm{~min}, 59^{\circ} \mathrm{C}$ for $1 \mathrm{~min}$, $72^{\circ} \mathrm{C}$ for $1 \mathrm{~min}$, and final extension at $72^{\circ} \mathrm{C}$ for $10 \mathrm{~min}$. The PCR products were resolved on $1 \%$ agarose gel.

For the detection of mature miRNAs, $1 \mu \mathrm{g}$ RNA extracted from cells was reverse transcribed to cDNA using M-MLV reverse transcriptase (Promega) and the primers were: miR145-RT, 5'-CTCAACTGGTGTCGTGGAGTCGGCAATTC AGTTGAGAGGGATTC-3' and U6-RT, 5'-GTCGTATCCA GTGCAGGGTCCGAGGTATTCGCACTGGATACGACA AAATATGGAAC-3', which can fold to a stem-loop structure. The cDNA was used for the amplification of mature miR-145 and an endogenous control U6, respectively, through PCR reaction. PCR primers were: $\mathrm{miR} 145-\mathrm{Fwd}, 5^{\prime}$-ACACTCCA GCTGGGCAGGTCAAAAGGGTCC-3' and U6-Fwd, 5'TGCGGGTGCTCGCTTCGGCAGC-3', which could ensure the specificity of the PCR products and reverse 5'-GGT GTCGTGGAGTCG-3', which was universal. PCR cycles were as follows: initial denaturation at $95^{\circ} \mathrm{C}$ for $5 \mathrm{~min}$, followed by 30 cycles of $94^{\circ} \mathrm{C}$ for $0.5 \mathrm{~min}, 56^{\circ} \mathrm{C}$ for $1 \mathrm{~min}, 72^{\circ} \mathrm{C}$ for $0.5 \mathrm{~min}$, and final extension at $72^{\circ} \mathrm{C}$ for $10 \mathrm{~min}$. The PCR products were resolved on $2 \%$ agarose gel.

TaqMan real-time PCR. The expression level of miR-145 was quantified by TaqMan microRNA real-time assay, which was performed by using the Applied Biosystems 7500 Sequence Detection System (Applied Biosystems). The expression of miRNA was defined from the threshold cycle $\left(C_{t}\right)$, and relative expression levels were calculated after normalization with reference to expression of U6 small nuclear RNA.

Cell growth assay. After transfection with vector control or miR-145-expressing plasmid, the cells were seeded into 96-well plates at 2000 cell/well. The cck-8 assay was used to determine relative cell growth according to the manufacturer's instructions. Data shown are representative of three independent experiments.

Luciferase activity assay. Dual luciferase vector pRL-TK and pGL3 were purchased from Promega. An oligonucleotide duplex containing the predicted binding site of miR-145 present in the 3'UTR of RTKN or mutant sequence was inserted after the luciferase gene of pRL-TK control vector. The oligonucleotide sequences used were as follows: RTKNwild-type sense, 5'-CTAGACTCGCTGGGACCTCCCTAA ACCCTTCCTGGAAGAAAACTGGAACCAACTCTGCCC TACCTCCCTGGC-3'; antisense, 5'-GGCCGCCAGGGAGG TAGGGCAGAGTTGGTTCCAGTTTTCTTCCAGGAA GGGTTTAGGGAGGTCCCAGCGAGT-3'. Mutant sense, 5'-CTAGACTCGCTGGGACCTCCCTAGGAAAGGTTTC
A

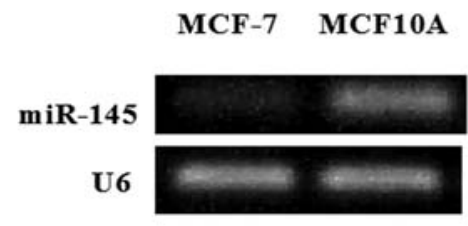

B

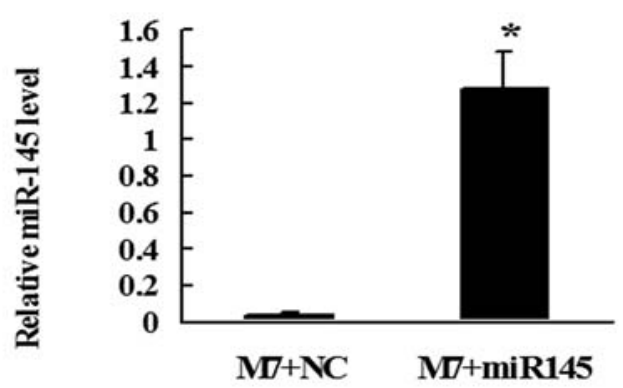

Figure 1. (A) Expression levels of miR-145 in MCF-7 and MCF10A were detected by stem-loop semi-quantitative RT-PCR assay. U6 snRNA was regarded as endogenous control. (B) Cells were harvested after EGFP expression was confirmed $48 \mathrm{~h}$ post-transfection, and total RNA was extracted, followed by TaqMan real-time PCR. miR-145 expression was normalized by U6 RNA (M7+NC, MCF-7 cells transfected with negative control vector; M7+miR145, MCF-7 cells transfected with miR-145-expressing vector). Three separate experiments were performed and the representative results are shown.

CGAGATTACTTTGGCCAACTCTGCCCTACCTCCCTG GC-3'; antisense, 5'-GGCCGCCAGGGAGGTAGGGCAGA GTTGGCCAAAGTAATCTCGGAAACCTTTCCTAGGGA GGTCCCAGCGAGT-3'. The plasmids were cotransfected with miR-145-expressing plasmid into MCF-7 cells. Luciferase activity was measured $24 \mathrm{~h}$ after transfection with the Promega Luciferase assay.

Western blot analysis. Forty-eight hours after transfection, MCF-7 cells were lyzed with RIPA lysis buffer and proteins were harvested. Proteins were resolved on an SDS denatured polyacrylamide gel and then transferred onto a nitrocellulose membrane. Antibody to RTKN (Abcam) and antibody to ß-actin (Santa Cruz Biotechnology, Inc., Santa Cruz, CA) were incubated with the blot overnight at $4^{\circ} \mathrm{C}$. Membranes were washed and incubated with respective secondary antibodies and were visualized by enhanced chemiluminescence (Millipore) according to the manufacturer's instructions. Shown are representative data from individual experiments that were repeated at least twice.

FACS analysis. Briefly, $5 \times 10^{5}$ of cells were harvested after transfection at indicated time. Cells were fixed overnight with $75 \%$ cold ethanol, washed twice with cold PBS, and then incubated in PBS buffer containing $50 \mu \mathrm{g} / \mathrm{ml}$ propidium iodide (PI) and $20 \mu \mathrm{g} / \mathrm{ml} \mathrm{RNase}$ A for $30 \mathrm{~min}$ at $37^{\circ} \mathrm{C}$. PI and forward light scattering were detected using a flow cytometer of the type FACSCalibur (Beckton-Dickinson USA) equipped with the ModFit LT software package.

Statistical analysis. Data are represented as means \pm SD of three independent experiments, each performed in triplicate. 
A

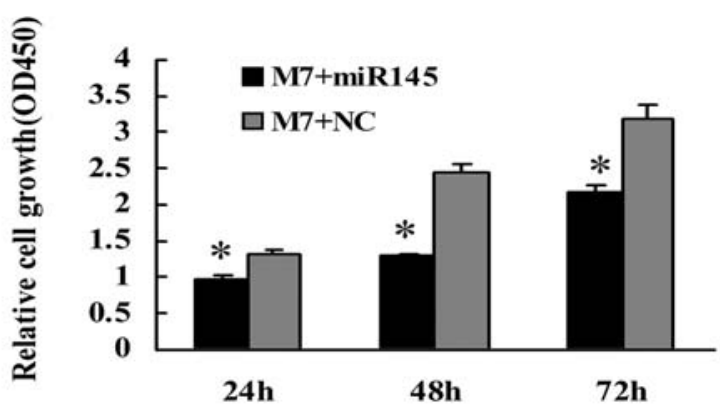

$\mathrm{B}$

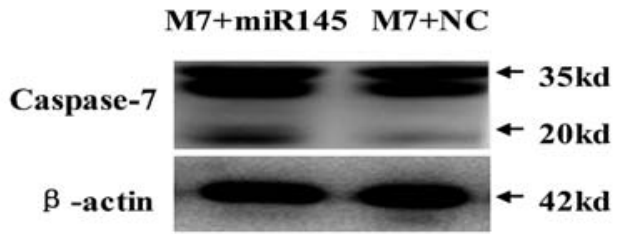

$\mathrm{C}$
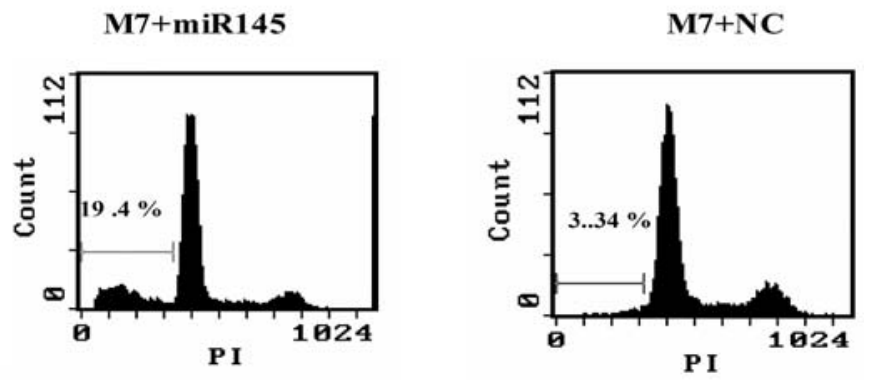

Figure 2. Growth inhibition and apoptosis induction by miR-145 overexpression in MCF-7 cells (A) in vitro cell growth analysis. MCF-7 cells were transfected with either vector control or miR-145-expressing plasmid. Relative cell growth was determined at the indicated times by cck-8 assay. Values are shown as mean \pm SD of three separate experiments. (B) Western blot analysis of apoptotic cell marker caspase-7. The molecular sizes of the intact and cleaved forms caspase-7 are indicated on the right. (C) Percentage of apopototic cells in the sub-G1 fraction was shown 48 h after transfection by FACS (M7+NC, MCF-7 cells transfected with negative control vector; M7+miR145, MCF-7 cells transfected with miR-145-expressing vector).

Statistical significance between treatment and control groups was analyzed using the Student's t-test. $\mathrm{P}<0.05$ was regarded as significant.

\section{Results}

Down-regulation of miR-145 in MCF-7 cells. Previous studies have demonstrated a significant down-regulation of miR-145 in several types of cancer, using stem-loop semi-quantitative RT-PCR assay as described (15), we found that MCF-7 cells express a significantly lower level of endogenous miR-145 than MCF10A, a normal human mammary epithelial cell line (Fig. 1A). miR-145 is derived from genomic sequences within $4.09 \mathrm{~kb}$ on chromosome 5 (5q32-33) (http://microrna.sanger. ac.uk/sequences). The locus of miR-145 coding gene is adjacent to $5 \mathrm{q} 31.1$. Since $5 \mathrm{q} 31.1$ is a well-known fragile site in human genome (http://www.genenames.org/) and in 11\% of sporadic breast cancers, 5q12-31 is deleted (16), it may contribute to the low expression of miR-145 found in MCF-7 cells. However this needs further exploration.

The silencing of miR-145 prompted us to investigate whether miR-145 functions as a tumor suppressor. To address this question, we changed the functional level of miR-145 in
MCF-7. We first constructed sense and antisense miR-145 sequences into a miRNA-like siRNA vector (17). This cloning step generated miR-145 expressing vectors that express mature sequences of miR-145. We use miR-145-expressing vector $(2.0 \mu \mathrm{g})$ in a 6-well plate. Twenty-four hours after transfection, the expression of a co-expressed fluorescent marker GFP was monitored using fluorescent microscope. miR-145 expression level was determined by TaqMan realtime PCR. As expected, the expression level of miR-145 was elevated by transfection of miR-145 expressing plasmid (Fig. 1B).

miR-145 inhibits MCF-7 cell growth and induces apoptosis in vitro. To investigate the functional significance of the overexpression of miR-145 in MCF-7, we performed CCK-8 assays in MCF-7 cells 24, 48 and $72 \mathrm{~h}$ after miR-145 transfection. As demonstrated in Fig. 2A, overexpression of miR-145 obviously impaired the cell growth. At the concentration of $2 \mu \mathrm{g}$ miR-145-expressing plasmids, the inhibition rate at 24,48 and $72 \mathrm{~h}$ after transfection was $26.4,47.2,31.9 \%$, respectively, compared to the vector control groups. This result was also in agreement with the previous reports that miR-145 could inhibit the growth of HeLa cell (18) and colon 
A

\begin{tabular}{|c|c|}
\hline Predicted consequential pairing of target region and miRNA & Pictar Score \\
\hline 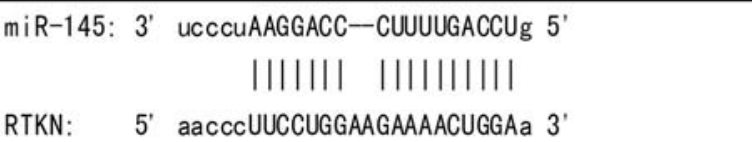 & 6.27 \\
\hline
\end{tabular}

B

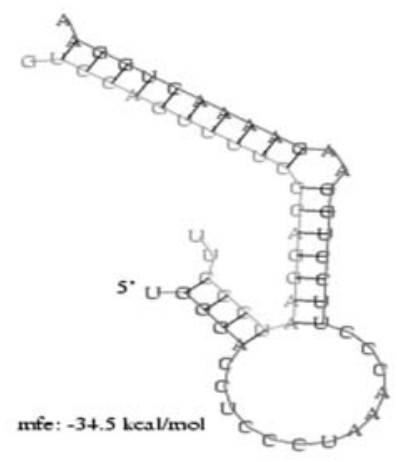

C
D
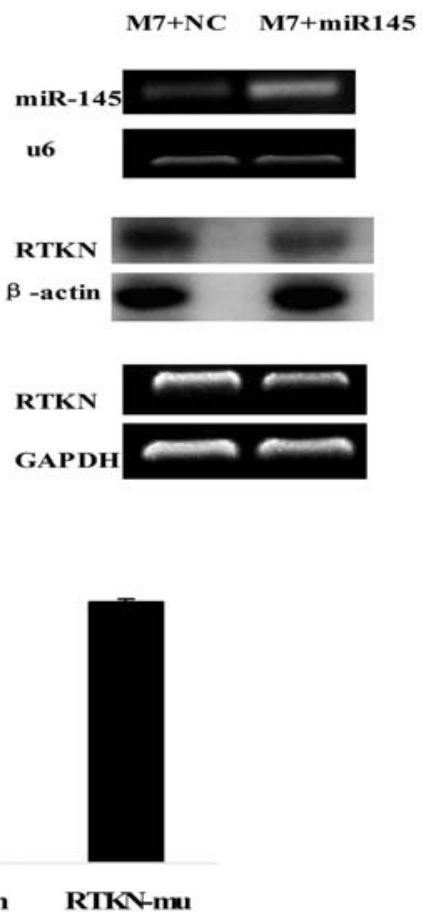

Figure 3. Effect of the putative miR-145 binding site derived from the RTKN 3'UTR on luciferase expression. (A) Sequence alignment of miR-145 with 3'UTR of RTKN. (B) Predicted RNA duplex structure of miR-145/RTKN targeting site generated by Pictar. (C) Relative luminescence of MCF-7 cells following transfection of miR-145 in conjunction with wild-type or mutated RTKN reporter constructs (RTKN-wt, MCF-7 cells transfected with a wild-type RTKN3'UTR reporter; RTKN-con, MCF-7 cells transfected with a control reporter that doesn't contain RTKN-3'UTR; RTKN-mu, MCF-7 cells transfected with an RTKN-3'UTR muted reporter). (D) miR-145 regulates RTKN protein expression and mRNA in MCF-7 cells. First group, expression levels of miR-145 in MCF-7 transfected with miR-145 expressing plasmid or control vector were detected by stem-loop semi-quantitative RT-PCR assay. Second group, RTKN protein was measured by Western blotting. B-actin was regarded as endogenous normalizer. Third group, RTKN mRNA was measured by semi-quantitative RT-PCR. GAPDH mRNA was regarded as endogenous normalizer.

carcinoma cell line (19). Thereby suggesting that downregulation of miR-145 may be, at least in part, involved in human cancer development. However, this suppressive effect on the cell growth was not caused by cell cycle arrest since there is no statistical difference between MCF-7 cells transfected with miR-145-expressing plasmid and control cells (data not shown). Western blot analysis for apoptosis-specific marker caspase-7 was then performed to examine whether apoptosis was induced by miR-145 overexpression. Significant induction of caspase-7 was indeed observed (Fig. 2B), and it was further confirmed by fluorescence activated cell sorter analysis, showing $19.4 \%$ of sub-G1 apoptotic population $48 \mathrm{~h}$ after transfection of miR-145-expressing plasmid, whereas transfection of cells with the control plasmid only had about 3-4\% of cells in sub-G1 fraction (Fig. 2C).

RTKN is a target of $m i R-145$. To understand the mechanisms by which miR-145 suppresses MCF-7 cell growth and induces apoptosis, we used several computational methods to help identify miR-145 targets in humans. Among the 100 targets predicted by both the TargetScan (20) and PicTar (21) search programs, RTKN was of particular interest, because it has been found to be overexpressed in many cancer-derived cell lines (22). More importantly, inhibition of RTKN sensitized cells to apoptotic stimuli (14). The prediction results are listed in Fig. 3A. The free energy of miR-145 binding to the RTKN targeting site was calculated using PicTar (mfe: $-34.5 \mathrm{kcal} /$ $\mathrm{mol}$ ), and the predicted structure is shown in Fig. 3B. To validate that the 3'UTR of RTKN is a direct target of miR-145, a 72-bp fragment within the 3'UTR that contains the putative miR-145 binding site was cloned into PRL-TK reporter vector, downstream of the luciferase gene. A control reporter vector was also developed in which the seed region of the miR-145 binding site was mutated. Co-transfection of MCF-7 cells with a wild-type RTKN-3'UTR (RTKN-wt) reporter and the miR-145-overexpressing plasmid resulted in a significant 
A

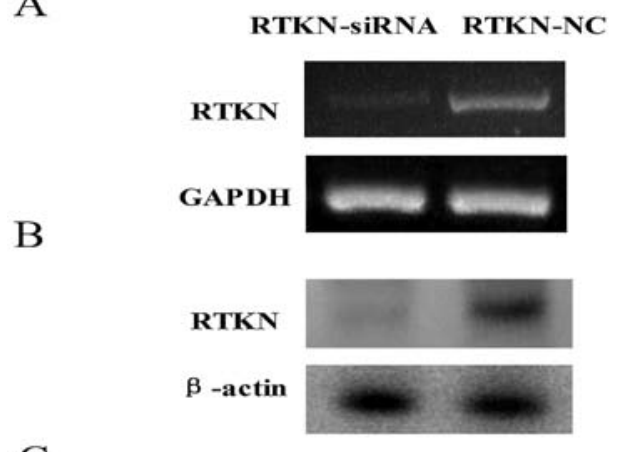

$\mathrm{C}$

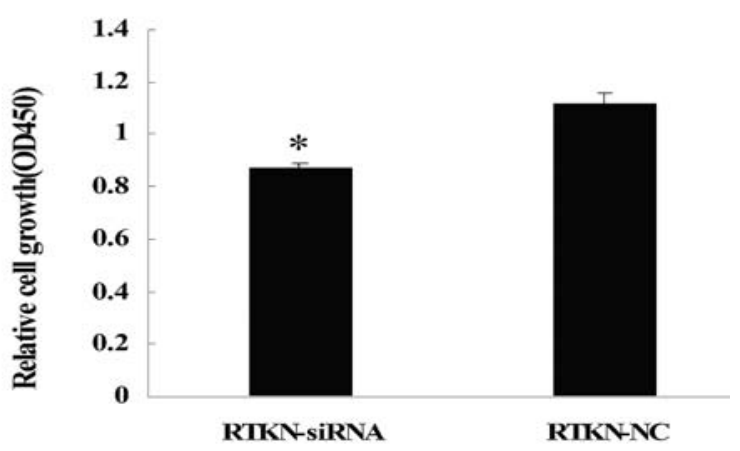

Figure 4. Elimination of RTKN expression inhibits MCF-7 cell growth. (A) Expression levels of RTKN in MCF-7 transfected with siRNA or negative control (con) analyzed by RT-PCR assay; and (B) Western blotting. (C) The cell viability was evaluated by CCK- 8 assay $48 \mathrm{~h}$ after siRNA transfection. Values are shown as mean \pm SD of three separate experiments (RTKN-siRNA, MCF-7 cells transfected with RTKN siRNA; RTKN-NC, MCF-7 cells transfected with negative control siRNA).

decrease in luminescence, whereas mutation of the RTKN miR-145 seed region (RTKN-mu) rendered the reporter construct insensitive to inhibition by miR-145 (Fig. 3C). Therefore, miR-145 is a direct inhibitor of RTKN.

miRNAs exert their effects by targeting the 3'UTR of the protein coding genes and thus induce translational repression and/or mRNA degradation (23). To assess whether miR-145 had a functional role in the down-regulation of endogenous RTKN expression, MCF-7 cells were transfected with miR-145 overexpressing plasmid. The effect of the transfection on the expression of 145 was monitored and validated by stem-loop semi-quantitative RT-PCR assay (Fig. 3D). By Western blot analysis, RTKN protein was down-regulated $48 \mathrm{~h}$ after transfection while there was no effect for the control transfection (Fig. 3D). On the other hand, the mRNA expression levels of RTKN were reduced slightly, when compared to the vectoronly control (Fig. 3D). Thus, miR-145 regulates RTKN protein expression and mRNA in MCF-7 cells. Taken together, we speculate that RTKN is a target of miR-145.

Elimination of RTKN expression by siRNA inhibits MCF-7 cell growth. We next ascertained whether reduction of RTKN levels might provide an explanation for the inhibition of cell growth observed following miR-145 overexpression. Small interfering RNA (siRNA) designed to target at RTKN was employed. Transfection of RTKN siRNA caused a $90 \%$ reduction in RTKN protein and mRNA level (Fig. 4A). We investigated a $18.6 \%$ cell growth inhibition $48 \mathrm{~h}$ after siRNA transfection (Fig. 4B). Hence, we conclude that RTKN is a functional target of miR-145. However, the effect of miR-145 overexpression on cell growth inhibition (47.2\%) is more significant than that of RTKN knockdown by RNAi. This may suggest that miR-145 targets other genes to exert its effect.

\section{Discussion}

Over the past five years, an ever-growing number of articles have been published describing a link between several forms of human cancer and the expression of microRNAs $(9,24-26)$. Deregulation (e.g., overexpression or loss of expression) of these so-called 'cancerous' miRNAs is associated with tumor initiation and progression (27). However, how these miRNA molecules contribute to the pathogenesis of cancer remains largely unknown. A detailed study on the biological functions of these miRNAs is therefore urgently desirable. Recently, some cancerous miRNAs have been characterized, such as let-7 $(28,29)$, miR-21 $(5,30)$ and miR-34 family $(31-33)$.

Down-regulation of miR-145 also has been found in several cancers, including colorectal cancer (34), B-cell lymphoma (35), cervical cancer $(36,37)$, hepatocellular carcinoma (38), ovarian cancer $(39)$ and breast cancer $(11,12)$. Kent and Mendell (40), in their review, list miR-145 as a tumor suppressor miRNA. Here, we found that miR-145 is downregulated in human cancer cell line MCF-7. Overexpression of miR-145 by plasmid inhibits MCF-7 cell growth and induces apoptosis. In none of these reported cases, however, were the targeted genes identified. Since the impact of cancerous miRNAs on cancer biology depends on the functions of the downstream targets they suppress, we need to uncover the targets of each miRNA. According to miRBase (December 2006), miR-145 has 1093 predicted targets in human and 890 in mouse. Up to date, the only validated miR-145 target is insulin receptor substrate-1 (IRS-1) (41). In this study, we identified RTKN as another target of miR-145. RTKN, the gene coding for the Rho effector, was shown to be expressed at a low level in normal cells and is overexpressed in many cancer-derived cell lines (14). RTKN overexpression conferred cell resistance to apoptosis induced by serum deprivation or treatment with sodium butyrate, and the increased resistance correlated to the level of RTKN. Conversely, reducing RTKN expression by small interfering RNAs greatly sensitized cells to apoptosis. RTKN may play a key role in tumorigenesis by conferring cells resistance to apoptosis (14). We found ectopic expression of miR-145 in MCF7 cells reduces RTKN protein and mRNA level. However, the effect of miR-145 overexpression on cell growth inhibition is more significant than that of RTKN knockdown by RNAi. This may suggest that miR-145 targets other genes to exert its effect. Additional studies are now needed for the identification and characterization of all miR-145 targets to gain a better comprehension of its function.

\section{Acknowledgements}

This work was supported from the ' 863 Projects' of Ministry of Science and Technology of P.R. China Grant (no. 2006AA02A109); from National Key Project for Basic 
Research of China (no. 001CB5099); Beijing Ministry of Science and Technology (no. D070507013507 01). R.C. Zhao is a Cheung Kong Scholar in P.R. China.

\section{References}

1. Bartel DP: MicroRNAs: genomics, biogenesis, mechanism, and function. Cell 116: 281-297, 2004

2. Croce CM and Calin GA: miRNAs, cancer, and stem cell division. Cell 122: 6-7, 2005.

3. Zhang B, Pan X, Cobb GP and Anderson TA: MicroRNAs as oncogenes and tumor suppressors. Dev Biol 302: 1-12, 2007.

4. Nam EJ, Yoon H, Kim SW, et al: MicroRNA expression profiles in serous ovarian carcinoma. Clin Cancer Res 14: 2690-2695, 2008.

5. Si ML, Zhu S, Wu H, Lu Z, Wu F and Mo YY: miR-21-mediated tumor growth. Oncogene 26: 2799-2803, 2007.

6. Tavazoie SF, Alarcón C, Oskarsson T, et al: Endogenous human microRNAs that suppress breast cancer metastasis. Nature 451: 147-152, 2008.

7. Calin GA, Cimmino A, Fabbri M, et al: MiR-15a and miR-16-1 cluster functions in human leukemia. Proc Natl Acad Sci USA 105: 5166-5171, 2008.

8. He L, Thomson JM, Hemann MT, et al: A microRNA polycistron as a potential human oncogene. Nature 435: 828-833, 2005.

9. Volinia S, Calin GA, Liu CG, et al: A microRNA expression signature of human solid tumors defines cancer gene targets. Proc Natl Acad Sci USA 103: 2257-2261, 2006.

10. Giacinti L, Claudio PP, Lopez M and Giordano A: Epigenetic information and estrogen receptor alpha expression in breast cancer. Oncologist 11: 1-8, 2006.

11. Sempere LF, Christensen M, Silahtaroglu A, et al: Altered MicroRNA expression confined to specific epithelial cell subpopulations in breast cancer. Cancer Res 67: 11612-11620, 2007.

12. Iorio MV, Ferracin M, Liu CG, et al: MicroRNA gene expression deregulation in human breast cancer. Cancer Res 65: 7065-7070 2005 .

13. Reid T, Furuyashiki T, Ishizaki T, et al: Rhotekin, a new putative target for Rho bearing homology to a serine/threonine kinase, PKN, and rhophilin in the rho-binding domain. J Biol Chem 271: 13556-13560, 1996.

14. Liu CA, Wang MJ, Chi CW, Wu CW and Chen JY: Rho/ Rhotekin-mediated NF- $\mathrm{KB}$ activation confers resistance to apoptosis. Oncogene 23: 8731-8742, 2004.

15. Chen C, Ridzon DA, Broomer AJ, et al: Real-time quantification of microRNAs by stem-loop RT-PCR. Nucleic Acids Res 33: e179, 2005

16. Tirkkonen M, Tanner M, Karhu R, Kallioniemi A, Isola J and Kallioniemi OP: Molecular cytogenetics of primary breast cancer by CGH. Genes Chromosomes Cancer 21: 177-184, 1998.

17. Du G, Yonekubo J, Zeng Y, Osisami M and Frohman MA: Design of expression vectors for RNA interference based on miRNAs and RNA splicing. FEBS 273: 5421-5427, 2006.

18. Wang X, Tang S, Le SY, Lu R, Rader JS, Meyers C and Zheng ZM: Aberrant expression of oncogenic and tumorsuppressive microRNAs in cervical cancer is required for cancer cell growth. PLoS ONE 3: e2557, 2008.

19. Akao Y, Nakagawa Y and Naoe T: MicroRNAs 143 and 145 are possible common onco-microRNAs in human cancers. Oncol Rep 16: 845-850, 2006.

20. Grimson A, Farh KK, Johnston WK, Garrett-Engele P, Lim LP and Bartel DP: MicroRNA targeting specificity in mammals: determinants beyond seed pairing. Mol Cell 27: 91-105, 2007.
21. Krek A, Grün D, Poy MN, et al: Combinatorial microRNA target predictions. Nat Genet 37: 495-500, 2005.

22. Fan J, Ma LJ, Xia SJ, et al: Association between clinical characteristics and expression abundance of RTKN gene in human bladder carcinoma tissues from Chinese patients. J Cancer Res Clin Oncol 131: 157-162, 2005.

23. He L and Hannon GJ: MicroRNAs: small RNAs with a big role in gene regulation. Nat Rev Genet 5: 522-531, 2004.

24. Calin GA and Croce CM: MicroRNA signatures in human cancers. Nat Rev Cancer 6: 857-866, 2006.

25. Lu J, Getz G, Miska EA, et al: MicroRNA expression profiles classify human cancers. Nature 435: 834-838, 2005.

26. Esquela-Kerscher A and Slack FJ: Oncomirs-microRNAs with a role in cancer. Nat Rev Cancer 6: 259-269, 2006.

27. Hwang HW and Mendell JT: MicroRNAs in cell proliferation, cell death, and tumorigenesis. Br J Cancer 94: 776-780, 2006.

28. Lee YS and Dutta A: The tumor suppressor microRNA let-7 represses the HMGA2 oncogene. Genes Dev 21: 1025-1030, 2007.

29. Johnson SM, Grosshans H, Shingara J, et al: RAS is regulated by the let-7 microRNA family. Cell 120: 635-647, 2005.

30. Meng F, Henson R, Wehbe-Janek H, Ghoshal K, Jacob ST and Patel T: MicroRNA-21 regulates expression of the PTEN tumor suppressor gene in human hepatocellular cancer. Gastroenterology 133: 647-658, 2007.

31. Chang TC, Wentzel EA, Kent OA, et al: Transactivation of miR-34a by 53 broadly influences gene expression and promotes apoptosis. Mol Cell 26: 745-752, 2007.

32. Corney DC, Flesken-Nikitin A, Godwin AK, Wang W and Nikitin AY: MicroRNA-34b and MicroRNA-34c are targets of p53 and cooperate in control of cell proliferation and adhesionindependent growth. Cancer Res 67: 8433-8438, 2007

33. Tazawa H, Tsuchiya N, Izumiya $M$ and Nakagama H: Tumorsuppressive miR-34a induces senescence-like growth arrest through modulation of the E2F pathway in human colon cancer cells. Proc Natl Acad Sci USA 104: 15472-15477, 2007.

34. Slaby O, Svoboda M, Fabian P, et al: Altered expression of miR-21, miR-31, miR-143 and miR-145 is related to clinicopathologic features of colorectal cancer. Oncology 72: 397-402, 2007.

35. Akao Y, Nakagawa Y, Kitade Y, Kinoshita T and Naoe T : Downregulation of microRNAs-143 and -145 in B-cell malignancies. Cancer Sci 98: 1914-1920, 2007.

36. Lui WO, Pourmand N, Patterson BK and Fire A: Patterns of known and novel small RNAs in human cervical cancer. Cancer Res 67: 6031-6043, 2007.

37. Martinez I, Gardiner AS, Board KF, Monzon FA, Edwards RP and Khan SA: Human papillomavirus type 16 reduces the expression of microRNA-218 in cervical carcinoma cells. Oncogene 27: 2575-2582, 2008

38. Varnholt H, Drebber U, Schulze F, Wedemeyer I, Schirmacher P, Dienes HP and Odenthal M: MicroRNA gene expression profile of hepatitis $\mathrm{C}$ virus-associated hepatocellular carcinoma. Hepatology 47: 1223-1232, 2008.

39. Iorio MV, Visone R, Di Leva G, et al: MicroRNA signatures in human ovarian cancer. Cancer Res 67: 8699-8707, 2007.

40. Kent OA and Mendell JT: A small piece in the cancer puzzle: microRNAs as tumor suppressors and oncogenes. Oncogene 25: 6188-6196, 2006.

41. Shi B, Sepp-Lorenzino L, Prisco M, Linsley P, De Angelis T and Baserga R: Micro RNA-145 targets the insulin receptor substrate-1 and inhibits the growth of colon cancer cells. J Biol Chem 282: 32582-32590, 2007. 